\title{
Lie group method and fractional differential equations
}

\author{
M. M. Alshamrania,*, H. A. Zedan ${ }^{b}$, M. Abu-Nawas ${ }^{a}$ \\ a Department of Mathematics, Faculty of Science, Northern Border University, Arar, Saudi Arabia. \\ ${ }^{b}$ Department of Mathematics, Faculty of Science, Kafrelsheikh University, Egypt.
}

Communicated by D. Baleanu

\begin{abstract}
In this paper, Lie group method is applied to investigate and solve some classes of nonlinear fractional differential equations. In addition, we use the obtained symmetries to induce exact solutions for the equations under consideration. (C)2017 All rights reserved.
\end{abstract}

Keywords: Lie group method, nonlinear partial differential equations, symmetry analysis.

2010 MSC: 22EXX, 17BXX, 20GXX.

\section{Introduction}

In the recent decades, many mathematicians have used the fractional differential equations to describe several natural phenomena in different branches of science such as: fluid mechanics, physics, chemistry and biology. Moreover, fractional differential equations can be used to investigate the process of these branches of science in complex irregular conditions, see for example [29]. Although a large number of mathematical physics methods have been developed to solve differential equations, the Lie group method is still the most efficient approach to look for the exact and the explicit solutions of nonlinear partial differential equations. It can be noted that some phenomena may depend on the previous time as well as the current time. In such situations, the theory of derivatives and integrals of fractional order can be used to model them, see $[15,21]$.

As the symmetry property is a natural phenomena, the equations that describe chemical, physical or biological processes must possess the symmetry properties. By using ordinary or partial differential equations, these processes can be described. The most common way to study the symmetry properties of these equations is the group analysis method.

Recently, a considerable number of researchers have investigated the symmetry properties of different types of equations using the Lie group analysis method. In [23], Wang and Xu investigated the symmetry properties of the time fractional KdV equations using the Lie group analysis method. In [22], Wang et al. studied the invariance properties of the time fractional fifth-order KdV equations using the Lie

\footnotetext{
${ }^{*}$ Corresponding author

Email addresses: malshomrani@hotmail.com (M. M. Alshamrani), hassanzedan2003@yahoo.com (H. A. Zedan), m. abunawas. math. nbu@gmail. com (M. Abu-Nawas)

doi:10.22436/jnsa.010.08.13
} 
group analysis method. Huang and Zhdanov, in [13], determined the maximal symmetry group and the corresponding optimal system of subgroups for the time fractional Harry-Dym equation using the Lie group analysis method. Moreover, in [28], Zedan and Alshamrani considered the (2+1)-dimensional higher-order Broer-Kaup System and used Lie symmetry analysis to determine the optimal systems for subalgebras and their corresponding solutions. In [7], the number of independent variables of the time fractional partial equations is reduced by using Lie analysis method. Furthermore, Wang and Xu in [24] performed the Lie group analysis method on the nonlinear perturbed Burgers equation and also on the nonlinear time fractional perturbed Burgers equation to construct the vector fields of these equations.

However, the interested reader can be also referred to the following relevant recent papers in the area of linear and nonlinear fractional partial differential equations and their use to describe a series of phenomena in applied sciences: $[1-3,5,14,16,27,30]$.

One can extend Lie groups and their infinitesimal generators to act on the space of independent variables, state variables and their derivatives (the state variables) up to an arbitrary finite order. Despite the fact that Lie symmetries were initially used to solve ordinary differential equations, they are also used to reduce systems of differential equations and to find simpler equivalent systems.

In the last few years, the fractional differential equations were heavily used in mathematical modeling of the processes of physics. It was not common to apply the symmetry properties for studying fractional differential equations. In [10], Gazizov et al. used Lie continuous groups for symmetry analysis of the fractional differential equations and suggested a formula for fractional derivatives.

In this paper, we use the Lie symmetry group method to investigate the following nonlinear system of fractional differential equations [31]:

$$
\begin{array}{ll}
\mathrm{D}^{\alpha} \mathrm{u}=\mathrm{u}, & 0<\alpha \leqslant 1, \\
\mathrm{D}^{\beta} v=2 \mathrm{u}^{2}, & 0<\beta \leqslant 1, \\
\mathrm{D}^{\gamma} z=3 u v, & 0<\gamma \leqslant 1,
\end{array}
$$

where $\alpha, \beta$, and $\gamma$ are real constants. Here $u, v$, and $z$ are functions of independent variable $x$, and $D^{\alpha} u$ is a fractional derivative of $u$ with respect to $x$, which can be of the following Riemann-Liouville type:

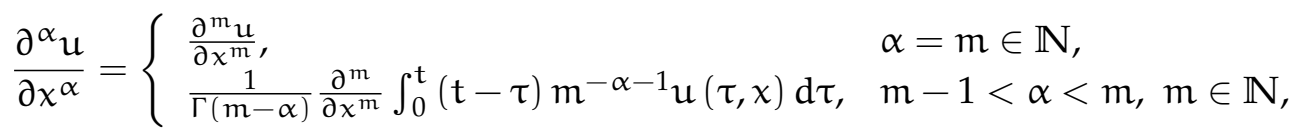

see $[8,12]$.

\section{Lie symmetry analysis for FPDs}

In this section, brief details of Lie symmetry analysis for FPDs with one independent variable and three dependent variables are presented. We consider a scalar time FPDEs of the following form:

$$
\begin{array}{ll}
\frac{\partial^{\alpha} u(x)}{\partial x^{\alpha}}=F_{1}\left(x, u, v, z, u_{x}, v_{x}, z_{x}, u_{x x}, \cdots\right), & \alpha>0, \\
\frac{\partial^{\beta} v(x)}{\partial x^{\beta}}=F_{2}\left(x, u, v, z, u_{x}, v_{x}, z_{x}, u_{x x}, \cdots\right), & \beta>0, \\
\frac{\partial^{\gamma} z(x)}{\partial x^{\gamma}}=F_{3}\left(x, u, v, z, u_{x}, v_{x}, z_{x}, u_{x x}, \cdots\right), & \gamma>0,
\end{array}
$$

where subscripts denote partial derivatives. Following [19, 20, 22], we assume that the above FPDE (2.1), is invariant under a one parameter $(\epsilon)$. Cosidering Lie theory, the construction of the symmetry group is equivalent to the determination of its infinitesimal transformations:

$$
\bar{\chi}=x+\epsilon \xi(x, u, v, z)+O\left(\epsilon^{2}\right),
$$




$$
\begin{aligned}
\bar{u} & =u+\epsilon \eta_{1}(x, u, v, z)+\mathrm{O}\left(\epsilon^{2}\right), \\
\bar{v} & =v+\epsilon \eta_{2}(x, u, v, z)+\mathrm{O}\left(\epsilon^{2}\right), \\
\bar{z} & =z+\epsilon \eta_{3}(x, u, v, z)+\mathrm{O}\left(\epsilon^{2}\right), \\
\frac{\partial^{\alpha} \bar{u}}{\partial \bar{x}^{\alpha}} & =\frac{\partial^{\alpha} u}{\partial x^{\alpha}}+\epsilon \zeta_{\alpha}^{0}+\mathrm{O}\left(\epsilon^{2}\right), \\
\frac{\partial^{\beta} \bar{v}}{\partial \bar{x}^{\beta}} & =\frac{\partial^{\beta} v}{\partial x^{\beta}}+\epsilon \zeta_{\beta}^{0}+\mathrm{O}\left(\epsilon^{2}\right), \\
\frac{\partial^{\gamma} \bar{z}}{\partial \bar{x} \gamma} & =\frac{\partial^{\alpha} z}{\partial x^{\alpha}}+\epsilon \zeta_{\gamma}^{0}+\mathrm{O}\left(\epsilon^{2}\right), \\
\frac{\partial \bar{u}}{\partial \bar{x}} & =\frac{\partial u}{\partial x}+\epsilon \zeta_{1}^{1}+\mathrm{O}\left(\epsilon^{2}\right),
\end{aligned}
$$

where $\xi, \eta_{1}, \eta_{2}$, and $\eta_{3}$ are infinitesimal and $\zeta_{1}^{1}, \zeta_{\alpha}^{0}, \zeta_{\beta}^{0}$, and $\zeta_{\gamma}^{0}$ are extended infinitesimal of order $1, \alpha, \beta$, and $\gamma$, respectively [9]. Infinitesimal generator takes the form:

$$
X=\xi \frac{\partial}{\partial x}+\eta_{1} \frac{\partial}{\partial u}+\eta_{2} \frac{\partial}{\partial v}+\eta_{3} \frac{\partial}{\partial z} .
$$

The following equation is the $\alpha$-th extended infinitesimal related to Rieman-Liouville fractional time derivative with (1.2):

$$
\zeta_{\alpha}^{0}=D_{\chi}^{\alpha}\left(\eta_{1}\right)-\alpha D_{x}(\xi) D_{\chi}^{\alpha} u-\sum_{n=1}^{\infty}\left(\begin{array}{c}
\alpha \\
n+1
\end{array}\right) D_{x}^{n+1}(\xi) I_{\chi}^{\alpha-n}(u),
$$

see $[10,11]$. Here the operator $D_{\chi}^{\alpha}$ stands for the total fractional derivative operator [17]. The first term $D_{\chi}^{\alpha}\left(\eta_{1}\right)$ in the right hand side of equation (2.3) can be rewritten as:

$$
\begin{aligned}
D_{x}^{\alpha}\left(\eta_{1}\right)= & \frac{\partial^{\alpha} \eta_{1}}{\partial x^{\alpha}}+\eta_{1 u} \frac{\partial^{\alpha} u}{\partial x^{\alpha}}-u \frac{\partial^{\alpha} \eta_{1 u}}{\partial x^{\alpha}}+\eta_{1 v} \frac{\partial^{\alpha} v}{\partial x^{\alpha}}-v \frac{\partial^{\alpha} \eta_{1 v}}{\partial x^{\alpha}}+\eta_{1 z} \frac{\partial^{\alpha} z}{\partial x^{\alpha}}-z \frac{\partial^{\alpha} \eta_{1 z}}{\partial x^{\alpha}} \\
& +\sum_{n=1}^{\infty}\left(\begin{array}{c}
\alpha \\
n
\end{array}\right) \frac{\partial^{n} \eta_{1 u}}{\partial x^{n}} I_{x}^{\alpha-n}(u)+\sum_{n=1}^{\infty}\left(\begin{array}{c}
\alpha \\
n
\end{array}\right) \frac{\partial^{n} \eta_{1 v}}{\partial x^{n}} I_{x}^{\alpha-n}(v)+\sum_{n=1}^{\infty}\left(\begin{array}{l}
\alpha \\
n
\end{array}\right) \frac{\partial^{n} \eta_{1 z}}{\partial x^{n}} I_{\chi}^{\alpha-n}(z)+\mu_{1},
\end{aligned}
$$

where

$$
\begin{aligned}
\mu_{1}= & \sum_{n=2}^{\infty} \sum_{m=2}^{n} \sum_{k=2}^{m} \sum_{r=0}^{k-1}\left(\begin{array}{l}
\alpha \\
n
\end{array}\right)\left(\begin{array}{l}
n \\
m
\end{array}\right)\left(\begin{array}{l}
k \\
r
\end{array}\right) \frac{1}{k !} \frac{x^{n-\alpha}}{\Gamma(n+1-\alpha)}\left\{[-u]^{r} \frac{\partial^{m}}{\partial x^{m}}\left(u^{k-r}\right) \frac{\partial^{n-m+k} \eta_{1}}{\partial x^{n-m} \partial u^{k}}\right. \\
& \left.+[-v]^{r} \frac{\partial^{m}}{\partial x^{m}}\left(v^{k-r}\right) \frac{\partial^{n-m+k} \eta_{1}}{\partial x^{n-m} \partial v^{k}}+[-z]^{r} \frac{\partial^{m}}{\partial x^{m}}\left(z^{k-r}\right) \frac{\partial^{n-m+k} \eta_{1}}{\partial x^{n-m} \partial z^{k}}\right\} .
\end{aligned}
$$

Thus, the $\alpha$-th extended infinitesimal given in (2.4) becomes

$$
\begin{aligned}
\zeta_{\alpha}^{0}= & \frac{\partial^{\alpha} \eta_{1}}{\partial x^{\alpha}}+\left(\eta_{1 u}-\alpha D_{x}(\xi)\right) \frac{\partial^{\alpha} u}{\partial x^{\alpha}}-u \frac{\partial^{\alpha} \eta_{1 u}}{\partial x^{\alpha}}+\eta_{1 v} \frac{\partial^{\alpha} v}{\partial x^{\alpha}} \\
& -v \frac{\partial^{\alpha} \eta_{1 v}}{\partial x^{\alpha}}+\eta_{1 z} \frac{\partial^{\alpha} z}{\partial x^{\alpha}}-z \frac{\partial^{\alpha} \eta_{1 z}}{\partial x^{\alpha}}+\mu_{1} \\
& +\sum_{n=1}^{\infty}\left[\left(\begin{array}{l}
\alpha \\
n
\end{array}\right) \frac{\partial^{n} \eta_{1 u}}{\partial x^{n}}-\left(\begin{array}{c}
\alpha \\
n+1
\end{array}\right) D_{x}^{n+1}(\xi)\right] I_{x}^{\alpha-n}(u) \\
& +\sum_{n=1}^{\infty}\left(\begin{array}{l}
\alpha \\
n
\end{array}\right) \frac{\partial^{n} \eta_{1 v}}{\partial x^{n}} I_{x}^{\alpha-n}(v)+\sum_{n=1}^{\infty}\left(\begin{array}{l}
\alpha \\
n
\end{array}\right) \frac{\partial^{n} \eta_{1 z}}{\partial x^{n}} I_{x}^{\alpha-n}(z) .
\end{aligned}
$$

$\zeta_{\beta}^{0}$ and $\zeta_{\gamma}^{0}$ can be calculated in a similar way. 


\section{Symmetry group analysis of the nonlinear system of fractional differential equations (1.1)}

We complete this section in the light of references $[4,6,18,25,26]$. Lie theory allows us to assume that the nonlinear system of fractional differential equations (1.1) is invariant under the one parameter transformations (2.2). Hence, the transformed equation can be given as:

$$
\begin{array}{ll}
\mathrm{D}^{\alpha} \mathrm{u}=\mathrm{u}, & 0<\alpha \leqslant 1, \\
\mathrm{D}^{\beta} v=2 \mathrm{u}^{2}, & 0<\beta \leqslant 1, \\
\mathrm{D}^{\gamma} z=3 u v, & 0<\gamma \leqslant 1,
\end{array}
$$

provided $u(x), v(x)$, and $z(x)$ satisfy (1.1). Determining equation will take the form:

$$
\begin{aligned}
\zeta_{\alpha}^{0}-\eta_{1}=0, & 0<\alpha \leqslant 1, \\
\zeta_{\beta}^{0}-4 u \eta_{1}=0, & 0<\beta \leqslant 1, \\
\zeta_{\gamma}^{0}-3 v \eta_{1}-3 u \eta_{2}=0, & 0<\gamma \leqslant 1,
\end{aligned}
$$

which depend on variables $\mathrm{I}_{x}^{\alpha-n} \mathrm{u}, \mathrm{I}_{x}^{\alpha-n} v, \mathrm{I}_{x}^{\alpha-n} z, \mathrm{I}_{x}^{\beta-n} \mathrm{u}, \mathrm{I}_{x}^{\beta-n} v, \mathrm{I}_{x}^{\beta-n} z, \mathrm{I}_{x}^{\gamma-n} \mathrm{u}, \mathrm{I}_{x}^{\gamma-n} v$, and $\mathrm{I}_{x}^{\gamma-n} z$ for $n=1,2, \ldots$ which are considered to be independent. The structure of (3.1) enables us to reduce it to a system of infinitely many linear FDEs. By substituting the expressions for $\zeta_{\alpha}^{0}, \zeta_{\beta}^{0}$, and $\zeta_{\gamma}^{0}$ given in (2.5) into (3.1) and equating various powers of derivatives of $u, v$, and $z$ to zero, we get the following an overdetermined system of linear equations:

$$
\begin{array}{r}
\xi_{u}=\xi_{v}=\xi_{z}=0, \\
\eta_{1 u}=\eta_{1 v v}=\eta_{1 z z}=\eta_{1 x v}=\eta_{1 x z}=0, \\
\eta_{2 u}=\eta_{2 v v}=\eta_{2 z z}=\eta_{2 x u}=\eta_{2 x z}=0, \\
\eta_{3 u}=\eta_{3 v v}=\eta_{3 z z}=\eta_{3 x u}=\eta_{3 x v}=0, \\
\frac{\partial^{\alpha} \eta_{1}}{\partial x^{\alpha}}-\left(\eta_{1 u}-\alpha \xi\right)(u)-u \frac{\partial^{\alpha} \eta_{1 u}}{\partial x^{\alpha}}-\eta_{1}=0, \\
\frac{\partial^{\beta} \eta_{2}}{\partial x^{\beta}}-\left(\eta_{2 v}-\beta \xi\right)\left(2 u^{2}\right)-u \frac{\partial^{\alpha} \eta_{u}}{\partial x^{\alpha}}-4 u \eta_{1}=0, \\
\frac{\partial^{\gamma} \eta_{3}}{\partial x^{\gamma}}-\left(\eta_{3 z}-\gamma \xi\right)(3 u v)-u \frac{\partial^{\alpha} \eta_{u}}{\partial x^{\alpha}}-3 v \eta_{1}-3 u \eta_{2}=0, \\
\left(\begin{array}{c}
\alpha \\
n
\end{array}\right) \frac{\partial^{n} \eta_{1 u}}{\partial x^{n}}-\left(\begin{array}{c}
\alpha \\
n+1
\end{array}\right) D_{x}^{n+1}(\xi)=0, \quad n \in \mathbb{N}, \\
\left(\begin{array}{c}
\beta \\
n
\end{array}\right) \frac{\partial^{n} \eta_{2 v}}{\partial x^{n}}-\left(\begin{array}{c}
\beta \\
n+1
\end{array}\right) D_{x}^{n+1}(\xi)=0, \quad n \in \mathbb{N}, \\
\left(\begin{array}{c}
\gamma \\
n
\end{array}\right) \frac{\partial^{n} \eta_{3 z}}{\partial x^{n}}-\left(\begin{array}{c}
\gamma \\
n+1
\end{array}\right) D_{x}^{n+1}(\xi)=0, \quad n \in \mathbb{N} .
\end{array}
$$

By solving this system, we conclude the following explicit form of infinitesimal:

$$
\xi=C_{1}, \quad \eta_{1}=C_{2} u, \quad \eta_{2}=2 C_{2} v, \quad \eta_{3}=3 C_{2} z,
$$

where $C_{1}$ and $C_{2}$ are arbitrary constants. Hence the infinitesimal operator becomes

$$
X=C_{1} \frac{\partial}{\partial x}+C_{2} u \frac{\partial}{\partial u}+2 C_{2} v \frac{\partial}{\partial v}+3 C_{2} z \frac{\partial}{\partial z} .
$$

All the similarity variables associated with the Lie symmetries can be derived by solving the following characteristic equation:

$$
\frac{d x}{C_{1}}=\frac{d u}{C_{2} u}=\frac{d v}{2 C_{2} v}=\frac{d z}{3 C_{2} z}
$$


The similarity functions associated with the infinitesimal generator $X$ take the following form:

$$
u=e^{k_{1} x} \varphi_{1}, \quad v=e^{2 k_{1} x} \varphi_{2}, \quad z=e^{3 k_{1} x} \varphi_{3},
$$

where $k_{1}=\frac{C_{2}}{C_{1}}$ is an arbitrary constant and

$$
\varphi_{2}=2^{1-\beta} k_{1}^{-\beta} \varphi_{1}^{2}, \quad \varphi_{3}=2^{1-\beta} 3^{-\gamma} k_{1}^{-(\beta+\gamma)} \varphi_{1}^{3} .
$$

Therefore, the solution for the nonlinear system of fractional differential equations (1.1) will take the following form:

$$
u=\varphi_{1} e^{k_{1} x}, \quad v=2^{1-\beta} k_{1}^{-\beta} \varphi_{1}^{2} e^{2 k_{1} x}, \quad z=2^{1-\beta} 3^{-\gamma} k_{1}^{-(\beta+\gamma)} \varphi_{1}^{3} e^{3 k_{1} x},
$$

where $k_{1}=\frac{C_{2}}{C_{1}}$ is an arbitrary constant.

\section{Conclusion}

The most common way to study the symmetry properties of ordinary or partial differential equations is the group analysis method. In this study, it has been shown that the method of Lie group analysis can be successfully extended to the investigation of symmetry properties of fractional differential equations and can be effectively used for constructing exact solutions of these equations.

\section{Acknowledgment}

This paper was financially supported by The Deanship of Scientific Research, Northern Border University, under the project no. 435-060-7. The authors would like to thank The Deanship of Scientific Research for their financial support.

\section{References}

[1] W. M. Abd-Elhameed, Y. H. Youssri, Spectral solutions for fractional differential equations via a novel Lucas operational matrix of fractional derivatives, Rom. J. Phys., 61 (2016), 795-813. 1

[2] M. A. Abdelkawy, M. A. Zaky, A. H. Bhrawy, D. Baleanu, Numerical simulation of time variable fractional order mobile-immobile advection-dispersion model, Rom. Rep. Phys., 67 (2015), 773-791.

[3] A. Agila, D. Baleanu, R. Eid, B. Irfanoglu, Applications of the extended fractional Euler-Lagrange equations model to freely oscillating dynamical systems, Rom. J. Phys., 61 (2016), 350-359. 1

[4] W. F. Ames, R. L. Anderson, V. A. Dorodnitsyn, E. V. Ferapontov, R. K. Gazizov, N. H. Ibragimov, S. R. Svirshchevskiĭ, CRC handbook of Lie group analysis of differential equations, Vol. 1, Symmetries, exact solutions and conservation laws. CRC Press, Boca Raton, FL, (1994). 3

[5] A. H. Bhrawy, A new spectral algorithm for time-space fractional partial differential equations with subdiffusion and superdiffusion, Proc. Rom. Acad. Ser. A Math. Phys. Tech. Sci. Inf. Sci., 17 (2016), 39-47. 1

[6] E. Buckwar, Y. Luchko, Invariance of a partial differential equation of fractional order under the Lie group of scaling transformations, J. Math. Anal. Appl., 227 (1998), 81-97. 3

[7] E. H. El Kinani, A. Ouhadan, Lie symmetry analysis of some time fractional partial differential equations, Int. J. Mod. Phys., 38 (2015), 8 pages. 1

[8] M. Gaur, K. Singh, On group invariant solutions of fractional order Burgers-Poisson equation, Appl. Math. Comput., 244 (2014), 870-877. 1

[9] R. K. Gazizov, A. A. Kasatkin, Construction of exact solutions for fractional order differential equations by the invariant subspace method, Comput. Math. Appl., 66 (2013), 576-584. 2

[10] R. K. Gazizov, A. A. Kasatkin, S. Y. Lukashchuk, Continuous transformation groups of fractional differential equations, (Russian) Vestnik USATU, 9 (2007), 125-135. 1, 2

[11] R. K. Gazizov, A. A. Kasatkin, S. Y. Lukashchuk, Symmetry properties of fractional diffusion equations, Phys. Scr., T136 (2009), 5 pages. 2

[12] R. K. Gazizov, A. A. Kasatkin, S. Y. Lukashchuk, Group-invariant solutions of fractional differential equations, Nonlinear science and complexity, Springer, Dordrecht, (2011), 51-59. 1 
[13] Q. Huang, R. Zhdanov, Symmetries and exact solutions of the time fractional Harry-Dym equation with Riemann-Liouville derivative, Phys. A, 409 (2014), 110-118. 1

[14] D. Kumar, J. Singh, D. Baleanu, A fractional model of convective radial fins with temperature-dependent thermal conductivity, Rom. Rep. Phys., 69 (2017), 13 pages. 1

[15] K. S. Miller, B. Ross, An introduction to the fractional calculus and fractional differential equations, A Wiley-Interscience Publication, John Wiley \& Sons, Inc., New York, (1993). 1

[16] K. Nouri, S. Elahi-Mehr, L. Torkzadeh, Investigation of the behavior of the fractional Bagley-Torvik and Basset equations via numerical inverse Laplace transform, Rom. Rep. Phys., 68 (2016), 503-514. 1

[17] K. B. Oldham, J. Spanier, The fractional calculus, Theory and applications of differentiation and integration to arbitrary order, With an annotated chronological bibliography by Bertram Ross, Mathematics in Science and Engineering, Academic Press [A subsidiary of Harcourt Brace Jovanovich, Publishers], New York-London, (1974). 2

[18] I. Podlubny, Fractional differential equations, An introduction to fractional derivatives, fractional differential equations, to methods of their solution and some of their applications, Mathematics in Science and Engineering, Academic Press, Inc., San Diego, CA, (1999). 3

[19] R. Sahadevan, T. Bakkyaraj, Invariant analysis of time fractional generalized Burgers and Korteweg-de Vries equations, J. Math. Anal. Appl., 393 (2012), 341-347. 2

[20] R. Sahadevan, T. Bakkyaraj, Invariant subspace method and exact solutions of certain nonlinear time fractional partial differential equations, Fract. Calc. Appl. Anal., 18 (2015), 146-162. 2

[21] S. G. Samko, A. A. Kilbas, O. I. Marichev, Fractional integrals and derivatives, Theory and applications, Edited and with a foreword by S. M. Nikol'skil, Translated from the 1987 Russian original, Revised by the authors, Gordon and Breach Science Publishers, Yverdon, (1993). 1

[22] G.-W. Wang, X.-Q. Liu, Y.-Y. Zhang, Lie symmetry analysis to the time fractional generalized fifth-order KdV equation, Commun. Nonlinear Sci. Numer. Simul., 18 (2013), 2321-2326. 1, 2

[23] G.-W. Wang, T.-Z. Xu, Symmetry properties and explicit solutions of the nonlinear time fractional KdV equation, Bound. Value Probl., 232 (2013), 13 pages. 1

[24] G.-W. Wang, T.-Z. Xu, Invariant analysis and explicit solutions of the time fractional nonlinear perturbed Burgers equation, Nonlinear Anal. Model. Control, 20 (2015), 570-584. 1

[25] G.-C. Wu, A fractional Lie group method for anonymous diffusion equations, Commun. Frac. Calc., 1 (2010), 27-31. 3

[26] G.-C. Wu, Lie group classifications and non-differentiable solutions for time-fractional Burgers Equation, Commun. Theor. Phys., 55 (2011), 1073-1076. 3

[27] X.-J. Yang, F. Gao, H. M. Srivastava, New rheological models within local fractional derivative, Rom. Rep. Phys., 69 (2017), 12 pages. 1

[28] H. Zedan, M. M. Alshamrani, A novel class of solutions for the $(2+1)$-dimensional higher-order Broer-Kaup system, Comput. Math. Appl., 69 (2015), 67-80. 1

[29] L. M. Zelenyi, A. V. Milovanov, Fractal topology and strange kinetics: from percolation theory to problems in cosmic electrodynamics, Phys. Usp., 47 (2004), 749-788. 1

[30] Y. Zhang, D. Baleanu, X.-J. Yang, New solutions of the transport equations in porous media within local fractional derivative, Proc. Rom. Acad. Ser. A Math. Phys. Tech. Sci. Inf. Sci., 17 (2016), 230-236. 1

[31] M. Zurigat, S. Momani, Z. Odibat, A. Alawneh, The homotopy analysis method for handling systems of fractional differential equations, Appl. Math. Model., 34 (2010), 24-35. 1 